Note additionnelle

\title{
DESCRIPTION DE LA MICROFILAIRE DE SPLENDIDOFILARIA GRETILLATI
}

\author{
Par Yves J. GOLVAN
}

Nous avons étudié les Microfilaires présentes dans le sang et sur les frottis de foie par apposition, récoltées au cours de l'autopsie d'une Perruche d'élevage à Tananarive (Agapornis cana Gmelin). Nous ne pouvons affirmer avec certitude qu'il s'agit bien de la Microfilaire de Splendidofilaria gretillati décrite dans la précédente note, car chez cet Oiseau on n'a pu trouver d'adultes. Cependant, cette hypothèse est très vraisemblable, puisque la Perruche de la même espèce chez laquelle on a trouvé le mâle type provenait du même élevage. L'étude a été faite sur des préparations colorées par le Giemsa selon la technique habituelle.

\section{Description}

Microfilaire présentant des courbures régulières, sans ondulations secondaires. Extrémité céphalique arrondie, queue relativement courte et obtuse, en « doigt de gant ».

\section{Dimensions moyennes mesurées sur 20 spécimens}

Longueur totale : $197 \mu$ (longueur minimum : $175 \mu$, longueur maximum : $220 \mu)$.

Largeur moyenne : $6,6 \mu$.

Anneau nerveux situé à $52 \mu$ de l'extrémité antérieure.

Pore excréteur situé à $74 \mu$ de l'extrémité antérieure.

Cellule excrétrice située à $78 \mu$ de l'extrémité antérieure.

Corps interne situé à $138 \mu$ de l'extrémité antérieure.

Première cellule rectale située à $155 \mu$ de l'extrémité antérieure.

Deuxième cellule rectale située à $159 \mu$ de l'extrémité antérieure.

Troisième cellule rectale située à $169 \mu$ de l'extrémité antérieure.

Quatrième cellule rectale située à $174 \mu$ de l'extrémité antérieure.

Pore anal situé à $183 \mu$ de l'extrémité antérieure. 
Espace céphalique extrêmement court, le premier noyau somatique est très proche de l'extrémité antérieure. "Mundgebilde 》 toujours bien colorés en rouge vermillon. Noyaux sous-cuticulaires et somatiques ovoïdes, tassés les uns contre les autres, colorés en violet rose. Les derniers noyaux atteignent l'extrémité caudale. Pore excréteur assez petit, n'interrompant pas la colonne nucléaire. Cellule excrétrice proche du pore excréteur. Corps interne non coloré par le Giemsa, apparaissant comme une masse sphéroïdale réfringente interrompant complètement la colonne nucléaire. Première cellule rectale (1) grosse, arrondie, placée assez loin en arrière du corps interne. Deuxième cellule rectale un peu plus petite que la première et proche d'elle. Troisième cellule rectale petite, distante de la deuxième. Quatrième cellule rectale petite, proche de la troisième. Pore anal toujours situé nettement en arrière de la quatrième cellule rectale, petit, n'interrompant pas la colonne nucléaire.

\section{Bibliographie}

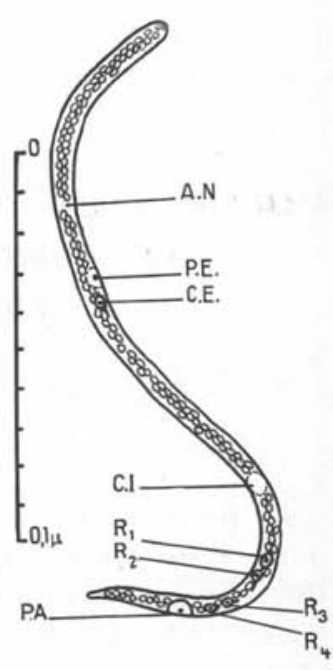

FIG. - Microfilaire de (?) Splendidofilaria gretillati. A.N.: anneaux nerveux ; P.E. : pore excréteur ; C.E. : cellule excrétrice ; C.I. : corps interne; R. 1 : première cellule rectale; R. 2 : deuxième cellule rectale ; R. 3 : troisième cellule rectale; R. 4 : quatrième c e 11 u le rectale ; P.A. : pore anal.

Anderson (R. C.), 1958. - Communication personnelle.

FENG (L. C.), 1936. - The development of Microfilaria malayi in Anopheles hyrcanus var. sinensis Weid. Chinese Med. Jl., Suppl., I, 345-367.

YAMADA (S.), 1927. - An experimental study of twenty-four species of japanese mosquitoes regarding their suitability as intermediate hosts for Filaria bancrofti Cobbold. Sci. Rep. Govt. Instit. Infect. Diseases, Tokyo, Imper. Univers., VI, 559-622.

(1) Note infrapaginale. - Dans nos précédentes notes sur l'anatomie de diverses Microfilaires, nous avons utilisé le terme de « cellules génitales pour désigner les quatre cellules placées en avant du pore anal et présentant des affinités tinctoriales particulières. Les travaux de Yamada (1927) et de Feng (1936), que complètent ceux actuellement en cours de Roy C. Anderson, montrent que cette interprétation est inexacte. Ces quatre cellules n'appartiennent pas à la liggnée germinale; elles ne donnent même pas les conduits génitaux de l'adulte. Elles donnent ultérieurement les parois du proctodeum. II est donc plus logique de les désigner sous le nom de « cellules rectales \$. Il est possible que ces cellules aient, chez la microfilaire, une fonction excrétrice (elles ont exactement les mêmes affinités tinctoriales que la cellule excrétrice proprement dite), fonction qu'elles perdent ensuite lorsque le système excréteur se développe et devient fonctionnel. 